\title{
Representing Excuses in Social Dependence Networks
}

\author{
Guido Boella ${ }^{1}$, Jan Broersen ${ }^{2}$, Leendert van der Torre ${ }^{3}$, and Serena Villata ${ }^{1}$ \\ 1 Dipartimento di Informatica, University of Turin, Italy \\ \{guido, villata\}@ai.unito.it \\ 2 Faculty of science, Universiteit Utrecht, The Netherlands \\ broersen@Cs.uu.nl \\ 3 CSC, University of Luxembourg, Luxembourg \\ leendert@vandertorre.com
}

\begin{abstract}
In this paper, we propose a representation of excuses in the context of multiagent systems. We distinguish five classes of excuses, taking as starting point both jurisprudential and philosophical studies about this topic, and we discuss their acceptance criteria. We highlight the following classes of excuses: epistemic excuses, power-based excuses, norm-based excuses, counts as-based excuses and social-based excuses and we represent them using social dependence networks. The acceptance criteria individuate those excuses which success in maintaining the trust of the other agents, e.g. in the context of social networks, excuses based on norms seem better than counts as-based ones in achieving this aim.
\end{abstract}

\section{Introduction}

One of the aims of multiagent systems is the representation of agents' social behaviour using notions as, for instance, trust, social laws, cooperation and so on. A notion which seems to have received less attention in this context is the concept of excuse. In jurisprudence, an excuse means to grant or obtain an exemption for a group of persons sharing a common characteristic from a potential liability. This means that the shown behavior cannot be approved but some excuse may be found, e.g. that the accused was a serving police officer or suffering from a mental illness. The notion of excuses is also treated in the context of philosophy, for example in Austin's "A Plea for Excuses" [1]. The New York Times of March 6, 1984 presents a new social theory about excuses. Particularly, they reported The garden variety - white lies that prevent hurt feelings ("Sorry, I can't make the party, I'm all tied up") - are so common, the research team says, because they are a social lubricant vital to the smooth operation of daily life. The presence of excuses in real life is everywhere thus the use of excuses as social lubricant should be extended from humans to intelligent agents of multiagent systems, especially where they are governed by social laws.

In this paper, we are interested in the distinction of the possible classes of excuses, representing them using social dependence networks in different ways. Moreover, using social dependence networks, we discuss what kinds of excuses seem more useful in convincing the other agents to trust you again. We address the following research questions: How to distinguish classes of excuses?, What are their acceptance criteria? and How to represent excuses in multiagent systems?. 
Norms and social laws can be represented as soft constraints, allowing for violations to be detected, instead of hard constraints that cannot be violated. Normally an agent fulfills its obligations, because otherwise its behavior counts as a violation that is sanctioned, and the agent dislikes sanctions. There are five categories of exceptions to this normal behavior which we call excuses. We distinguish these five classes of excuses considering the common human behaviours and the sources of the excuses. This classification seems the simplest one allowing anyway a certain variety of representation. The discussion of the acceptance criteria of excuses is strictly related to the way of representing them, by means of social dependence networks. First, epistemic excuses such as I did not know, I was not aware, I forgot. Second, excuses based on power such as I did not have the ability, opportunity or power to do it, others prevented me from doing it. Third, excuses based on normative conflicts such as there was another more important norm. Fourth, excuses based on difference of opinion on counts-as norms, such as I did comply, my act did not count as a violation. Finally, the common excuse Everybody does it.

We propose to use the methodology of dependence networks to represent these classes of excuses in multiagent systems' theory. The dependency modeling, used in software design by the TROPOS methodology [4], represents the multiagent system by means of goal-based dependencies and norm-based dependencies [10]. The advantage in using this methodology consists particularly in the representation of excuses like $E v$ erybody does it which are really difficult to be represented, for example in deontic logic. As in TROPOS, each agent has a dependence network representing its world knowledge and the network explicitly represents agents' excuses.

The reminder of the paper is as follows. In Section 2, we present the notion of excuses in philosophy and jurisprudence while section 3 describes the dependency modeling. In Section 4, we propose the five classes of excuses and their representation using social dependence networks. Related work and conclusions end the paper.

\section{Excuses in Social Sciences and Jurisprudence}

The word 'excuses' is strictly connected to terms such as 'plea', 'defence' and 'justification'. The main question sociologists and psychologists try to answer is When are 'excuses' proffered?. As described by Austin [1], the situation is one where someone is accused of having done something, or where someone is said to have done something which is bad, wrong, inept, unwelcome, or in some other of the numerous possible ways untoward. Thereupon he, or someone on his behalf, will try to defend his conduct or to get him out of it. One way of going about this is to admit flatly that he, X, did do that very thing, A, but to argue that it was a good thing, or the right or sensible thing, or a permissible thing to do, either in general or at least in the special circumstances of the occasion. To take this line is to justify the action, to give reason for doing it: not to say, to brazen it out, to glory in it, or the like. A different way of going about it is to admit that it was not a good thing to have done, but to argue that it is not quite fair or correct to say baldly ' $\mathrm{X}$ did A'. We may say it is not fair just to say $\mathrm{X}$ did it; perhaps he was under somebody's influence, or was nudged. A usual problem with excuses consists in their distinction from justifications. The two certainly can be confused. Let us consider the 
following example by Austin [1]. You dropped the tea-tray: Certainly, but an emotional storm was about to break out: or, Yes, but there was a wasp. In each case the defence insists on a fuller description of the event in its context but the first is a justification while the second is an excuse.

In jurisprudence [7], an excuse or justification is a form of immunity that must be distinguished from an exculpation. On the one hand, an exculpation is a defense in which the defendant argues that despite the fact that he has done everything to constitute the crime and so, in principle already has guilt for those actions or a liability to compensate the victim, he should be exculpated because of the special circumstances said to operate in favor of the defendant at the time the law was broken. On the other hand, a justification describes the quality of the act, whereas an excuse relates to the status or capacity (or lack of it) in the accused. To be excused from liability means that although the defendant may have been a participant in the sequence of events leading to the prohibited outcome, no liability will attach to the particular defendant because he belongs to a class of person exempted from liability. For instance, members of the armed forces may be granted a degree of immunity for causing prohibited outcomes while acting in the course of their official duties, e.g. for an assault or trespass to the person caused during a lawful arrest. Other excuses may due to a particular status and capacity. Finally, others may escape liability because the quality of their actions satisfied a general public good.

\section{Dependency Modeling}

Our model is a directed labeled graph whose nodes are instances of the metaclasses of the metamodel, e.g., agents, goals, and whose arcs are instances of the metaclasses representing relationships between them such as dependency and conditional dependency. In a multiagent system, since an agent is put into a system that involves also other agents, he can be supported by the others to achieve his own goals if he is not able to do them alone. In order to define these relations in terms of goals and powers, we adopt, as said, the methodology of dependence networks [8]. In this model, an agent is described by a set of prioritized goals, and there is a global dependence relation that explicates how an agent depends on other agents for fulfilling its goals. For example,

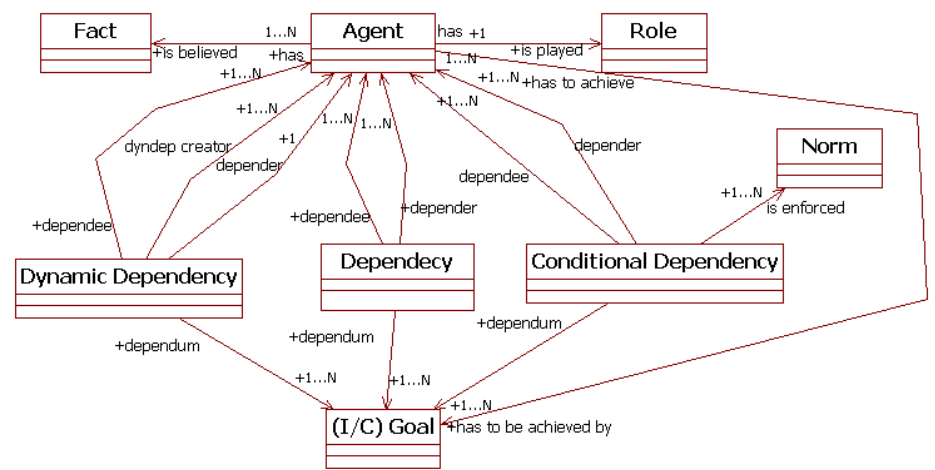

Fig. 1. The UML class diagram specifying the main concepts of the metamodel 
$\operatorname{dep}(\{a, b\},\{c, d\})=\left\{\left\{g_{1}, g_{2}\right\},\left\{g_{3}\right\}\right\}$ expresses that the set of agents $\{a, b\}$ depends on the set of agents $\{c, d\}$ to see to their goals $\left\{g_{1}, g_{2}\right\}$ or $\left\{g_{3}\right\}$. A definition of dependence networks is provided in [5].

The introduction of norms in dependence networks is based on the necessity to adapt the requirements analysis phases to model norm-based systems. An example of this kind of application consists in the introduction of obligations in virtual Grid-based organizations where obligations are used to enforce the authorization decisions. The UML diagram of Figure 1 provides a unified vision of the concepts of the ontology representing our conceptual metamodel. We define a normative view to describe the normative system, as follows:

Definition 1 (Normative View). Let the institutional view $\langle R L, I F, R G, X$, igoals : $R L \rightarrow 2^{R G}$, iskills $: R L \rightarrow 2^{X}$, irules $\left.: 2^{X} \rightarrow 2^{I F}\right\rangle$, the normative view is a tuple $\langle A, R G, N$, oblig, sanct, ctd $\rangle$ where:

- A is a set of agents, RG is a set of institutional goals, $N$ is a set of norms;

- the function oblig : $N \times A \rightarrow 2^{R G}$ is a function that associates with each norm and agent, the institutional goals the agent must achieve to fulfill the norm. Assumption: $\forall n \in N$ and $a \in A$, oblig $(n, a) \in \operatorname{power}(\{a\})$ 2.

- the function sanct $: N \times A \rightarrow 2^{R G}$ is a function that associates with each norm and agent, the institutional goals that will not be achieved if the norm is violated by agent a. Assumption: for each $B \subseteq A$ and $H \in \operatorname{power}(B)$ that $\left(\cup_{a \in A} \operatorname{sanct}(n, a)\right) \cap H=\emptyset$.

- the function ctd : $N \times A \rightarrow 2^{R G}$ is a function that associates with each norm and agent, the institutional goals that will become the new goals the agent a has to achieve if the norm is violated by a. Assumption: $\forall n \in N$ and $a \in A, \operatorname{ctd}(n, a) \in$ power $(\{a\})$.

We relate norms to goals following two directions. First, we associate with each norm $n$ a set of institutional goals oblig $(n) \subseteq R G$. Achieving these normative goals means that the norm $n$ has been fulfilled; not achieving these goals means that the norm is violated. Second, we associate with each norm a set of institutional goals $\operatorname{sanct}(n) \subseteq R G$ which will not be achieved if the norm is violated and it represents the sanction associated with the norm. Third, we associate with each norm (primary obligation) another norm (secondary obligation) represented by a set of institutional goals $\operatorname{ctd}(n) \subseteq R G$ that have to be fulfilled if the primary obligation is violated. Dependence networks representing norms are called conditional dependence networks and they are defined as follows:

\section{Definition 2 (Conditional Dependence Networks (CDN)).}

A conditional dependence network is a tuple $\langle A, R G$, cdep, odep, sandep, ctddep $\rangle$ where:

- A is a set of agents and RG is a set of institutional goals;

- cdep : $2^{A} \times 2^{A} \rightarrow 2^{2^{R G}}$ is a function that relates with each pair of sets of agents all the sets of goals on which the first depends on the second.

\footnotetext{
${ }^{1}$ irules associates sets of institutional actions with the sets of institutional facts to which they lead.

${ }^{2}$ Power relates each agent with the goals it can achieve.
} 
- odep : $2^{A} \times 2^{A} \rightarrow 2^{2^{R G}}$ is a function representing a obligation-based dependency that relates with each pair of sets of agents all the sets of goals on which the first depends on the second.

- sandep $\subseteq\left(O B L \subseteq\left(2^{A} \times 2^{A} \times 2^{2^{R G}}\right)\right) \times\left(S A N C T \subseteq\left(2^{A} \times 2^{A} \times 2^{2^{R G}}\right)\right)$ is a function relating obligations to the dependencies which represent their sanctions. Assumption: $S A N C T \in$ cdep and $O B L \in$ odep.

- ctddep $\subseteq\left(O B L_{1} \subseteq\left(2^{A} \times 2^{A} \times 2^{2^{R G}}\right)\right) \times\left(O B L_{2} \subseteq\left(2^{A} \times 2^{A} \times 2^{2^{R G}}\right)\right)$ is a function relating obligations to the dependencies which represent their secondary obligations. Assumption: $O B L_{1}, O B L_{2} \in$ odep and $O B L_{1} \cap O B L_{2}=\emptyset$.

\section{Representing Excuses in Dependence Networks}

\subsection{Classes of Excuses}

In this section we answer the research question: How to distinguish classes of excuses?. We highlight five classes of excuses: epistemic excuses, power-based excuses, norm-based excuses, counts as based excuses, social based excuses. These classes take into account different aspects of the social system from knowledge representation to norms and social laws. Particularly, the description of the classes of excuses is as follows:

1. Epistemic excuses: this class of excuses is related to the agents' knowledge. The distinction between epistemic excuses and moral ones consists in its relation with knowledge: an epistemic excuse transforms a true belief into knowledge. An example of this class of excuses consists in the following sentences: "I did not know, I was not aware, I forgot". Their peculiarity is the absence of knowledge which would make you able to see to your obligations or goals.

2. Power-based excuses: this class of excuses is based on an absence of the power to see to the obligation/goal the agent has to see to. An example of this class of excuses consists in the following sentences: "I did not have the ability, opportunity or power to do it, others prevented me from doing it". Their peculiarity is the absence of skills of the agent to perform the goal.

3. Norm-based excuses: this class of excuses is based on the presence of a norm with an higher priority which prevents the agent to see to its goals/obligations. An example of this class of excuses consists in the following sentence: "There was another more important norm I have to follow first". The peculiarity of this class of excuses consists in the presence of a preference order among the obligations of the agent.

4. Counts as-based excuses: this class of excuses is based on the idea that the excuse is about the fact that a violation did not occur because it did not count as one. An example of this class of excuses consists in the following sentence: "I did comply, my act did not count as a violation". This class of excuses could be seen as a sort of subclass of the epistemic class of excuses.

5. Social-based excuses: this class of excuses is based on society's common behaviours. The usual excuse of this kind is "Everybody does it". 


\subsection{Excuses in Dependence Networks}

In this section we answer the research question: How to represent excuses in multiagent systems?. The representation of excuses using the dependency modeling is presented by means of a semiformal language of visual modeling where the goal-based dependency is depicted as a plain arrowed line and the obligation-based dependency is depicted as a dashed arrowed line. The label of the arrow is the goal on which the dependency is based (normative goals for obligation-based dependencies and material goals for goalbased dependencies).

As in TROPOS [4], each agent has its own dependence network which represents his view of the systems' stakeholders. Epistemic excuses are represented by means of these multiple dependence networks as depicted in Figure 2] In this figure, we show two kinds of epistemic excuses, the first one related to a goal-based dependency and the second one related to an obligation-based dependency. Agent $a$ has his dependence network (the first square titled a) and it is involved as depender (see Figure 1 for the notion of depender) only in one norm-based dependency with agent $d$ but, as can be noted observing the other dependence networks, $a$ is also involved in a goal-based dependency with agent $b$. In this example, the excuse comes from the depender to convince the dependee, for instance if we see agent $a$ as a node of a Grid network having to store a file too big for its memory he can say that he has not this goal in order to not be dependent on agent $b$. In the second example, agent $a$ depends for a normative goal on agent $d$ but $d$ does not have in its dependence network this obligation-based dependency. In this case, the excuse comes from the dependee to convince the depender since the dependee does not want to support the depender in achieving its goal.

The representation of power-based excuses is achieved by means of absence of goalbased and obligation-based dependencies and it is connotated by grey arrows which represent a lack of power in terms of lacking skills. In Figure 3, agent $a$ depends on agent $b$ for achieving its goal but $b$ has the excuse that it has not the power to achieve this goal for agent $a$. A second case of this kind of excuses' representation is provided by the dependence network of agent $d$ which depends on itself for achieving its goal, for instance node $d$ has to run a computation and it is able to do it alone, but it can say that it does not have the power to do it, in order to have an help by the other agents. Note that

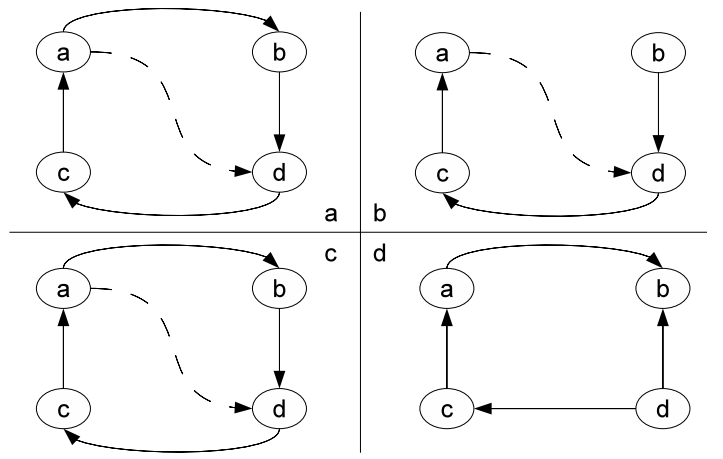

Fig. 2. Epistemic excuses 


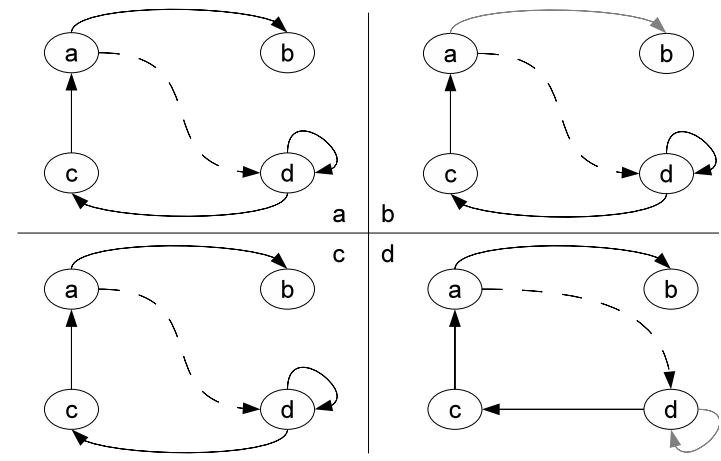

Fig. 3. Power-based excuses

the other agents of the system have in their dependence networks this self-dependence as an "active" one, since they "know" that agent $d$ is able to achieve its goal, thus this is an excuse.

Norm-based excuses are represented by means of the addition to the label not only of the goal on which the dependency is based, but also the priority on agents' goals. The formal addition of goal-based preferences is presented in [10]. In Figure 4 we have two possible cases of norm-based excuses. The first one involves agents $a, b$ and $d$. Agent $a$ depends on agent $b$ for normative goal $g 1$ and on agent $d$ for normative goal $g 2$. Agent $a$ prefers the dependency on $b$ than the one on $d$ so that in its dependence network $g 1$ is preferred over $g 2$. Agent $b$ agrees with $a$ while agent $d$ "thoughts" that for $a, g 2$ is preferred over $g 1$. Agent $a$ has an excuse for agent $d$ regarding the priority of normbased dependencies. The second case we highlight involves agents $b, c$ and $d$. Agent $d$ depends both on $b$ and $c$ respectively for normative goals $g 3$ and $g 4$. The difference consists in the preferences of agent $b$ which is the dependee of two norm based dependencies. Node $b$ can say to node $d$ that $g 1$ has the priority on $g 3$, so that it cannot achieve the normative goal $g 3$.

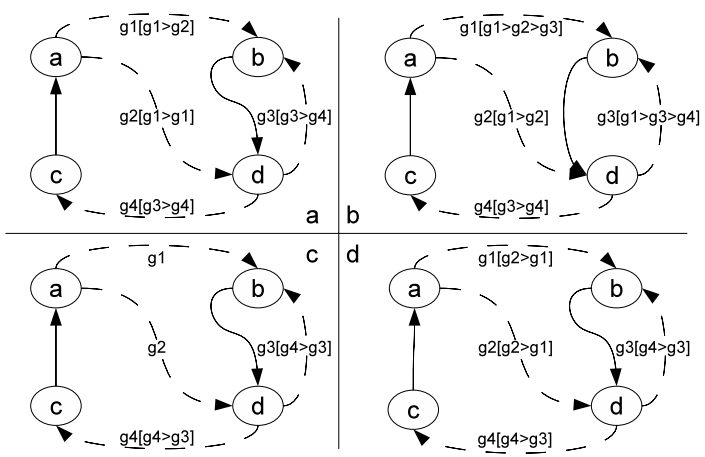

Fig. 4. Norm-based excuses 


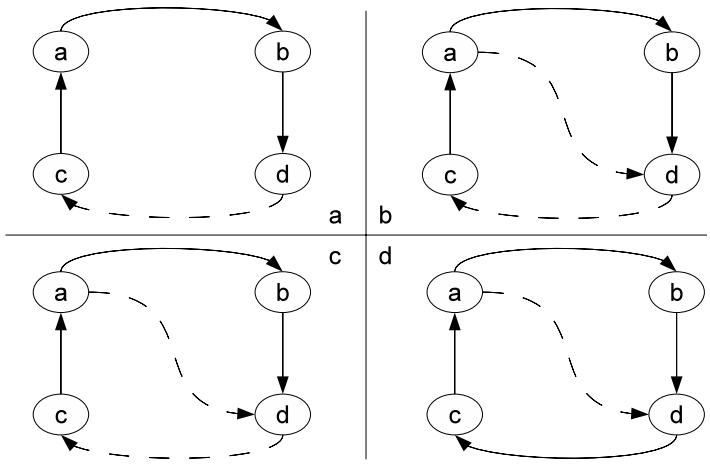

Fig. 5. Counts as-based excuses

Counts as based excuses are represented similarly to epistemic excuses. In Figure 5 counts as excuses are represented by the dependency between agent $d$ and agent $c$. Concerning agent $d$, its dependency on agent $c$ is a common one so that not achieving it does not mean a violation while for all the other agents this is an obligation-based dependency. The excuse of agent $d$, in the case this goal is not achieved, is that this does not count as a violation since it is not a normative goal.

Social-based excuses are the most difficult to be represented since they are summarized by sentences like "Everybody does it!". We represent this class of excuses by self-dependencies in the mind of the agent which uses this excuse since it sees that every agent is able in doing, for instance $g 1$, by itself. In Figure 6, agent $a$ is the agent using the excuse while, as shown by the dependence networks of the other agents of the system, it is not true that the other agents achieve $g 1$ alone since all of them depend on $d$ for $g 1$. This class of excuses cannot be directly represented using logical formalisms such as deontic logic while, using the dependency modeling technique, it is represented using self-dependencies on norms.

Concerning the acceptance criteria, we can fix the following points. Dependence networks represent how agents need each other to reach their goals. Agents need each

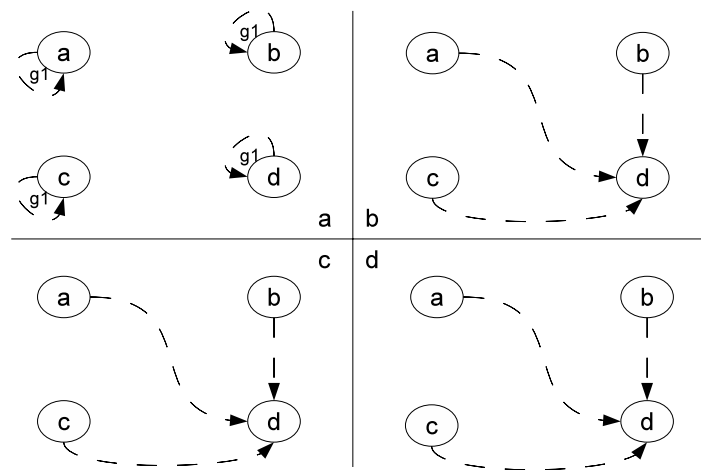

Fig. 6. Social-based excuses 
other and make promises, which can be seen as a kind of contract, to each other. In some cases, something goes wrong and some agent does not deliver. He is not immediately expelled if he has a good excuse. We can note that not knowing seems a bad excuse in these contexts, especially if there are explicit contracts. Higher authority, such as the preference order between norms, might be a good excuse, although agents should not make to much promises. Counts-as excuses seem a little far-fetched here. 'Everybody does it' is an excuse, but not a very constructive one in this context. One agent can say to the others: "I break my promise because you broke yours", and, besides, nobody holds his promises around here but then the dependency causes the system to halt.

\section{Related Work}

As far as we know, the only approach in the field of multiagent systems related to the topic of excuses consists in the definition of the notion of forgiveness by Vasalou et al. [9]. Online offences are generally considered as frequent and intentional acts performed by a member with the aim to deceive others. However, an offence may also be unintentional or exceptional, performed by a benevolent member of the community. Vasalou et al. [9] examine whether a victim's decrease in trust towards an unintentional or occasional offender can be repaired in an online setting, by designing and evaluating systems to support forgiveness. In their experiments, an apology from the offender restores the victim's trust only if the offender cooperates in a future interaction; it does not alleviate the trust breakdown immediately after it occurs. By contrast, the forgiveness component restores the victims trust directly after the offence and in a subsequent interaction. Forgiveness is a prosocial process during which negative motivations towards the offender are reduced and replaced with positive motivations [6]. It should be noted that, while a certain violation may be forgiven, other past behaviours may still impede one's trust towards another. Forgiveness mediates and resolves conflicts to sustain healthy long-term relationships [6]. A forgiving attitude promotes cooperation when compared to a vengeful outlook [2].

\section{Summary}

In this paper, we highlight five classes of excuses: epistemic excuses, power-based excuses, norm-based excuses, counts as-based excuses and social-based excuse. This classification seems to cover the main kinds of excuses, anyway it is possible that an excuse falls into two classes at the same time, such as for example epistemic excuses and counts as ones since the last one could be seen as a subclass of the former. The classification can be used to define different representations of the classes of excuses and consequently, to define different acceptance criteria due to the chosen representation. We represent excuses in multiagent systems using the methodology of social dependence networks. This methodology has the advantage, particularly in contrast with logical approaches, due to the representation of the last kind of excuses such as the common excuses "Everybody does it!". While, on the one side, these excuses are represented using dependence networks, as depicted in Figure 6, on the other side, they cannot be represented in deontic logic in a direct way. Moreover, dependence networks are used 
in design methodologies like the TROPOS one [4] and they are abstract, so they can be used, for example, for conceptual modeling, simulation, design and formal analysis.

Concerning future works, we are studying how excuses could help the formation of coalitions [3] acting as social lubricant. The idea is that each coalition has to maintain its stability but certain agents' behaviours may destroy this stability, such as laying to the other members of the coalition, decreasing in this way the level of trust inside it. Excuses can be seen as a social lubricant able to maintain, always due to time limits, coalition's stability despite negative behaviours of one or more of its members. Moreover, this study has to consider the addition of the notion of trust in dependence networks.

\section{References}

1. Austin, J.L.: A plea for excuses. Philosophical Papers (1961)

2. Axelrod, R.: The Evolution of Cooperation. Basic Books, New York (1984)

3. Boella, G., van der Torre, L., Villata, S.: Social viewpoints for arguing about coalitions. In: Bui, T.D., Ho, T.V., Ha, Q.-T. (eds.) PRIMA 2008. LNCS (LNAI), vol. 5357, pp. 66-77. Springer, Heidelberg (2008)

4. Bresciani, P., Perini, A., Giorgini, P., Giunchiglia, F., Mylopoulos, J.: Tropos: An agentoriented software development methodology. Autonomous Agents and Multi-Agent Systems 8(3), 203-236 (2004)

5. Caire, P., Villata, S., Boella, G., van der Torre, L.: Conviviality masks in multiagent systems. In: Padgham, L., Parkes, D.C., Müller, J., Parsons, S. (eds.) AAMAS (3), pp. 1265-1268. IFAAMAS (2008)

6. McCullough, M., Worthington, E.L., Rachal, K.C.: Interpersonal forgiving in close relationships. Journal of Personality and Social Psychology 73, 321-336 (1997)

7. Robinson, P.H.: Criminal law defenses: A systematic analysis. Columbia Law Review (199) (1982)

8. Sichman, J.S., Conte, R.: Multi-agent dependence by dependence graphs. In: AAMAS, pp. 483-490. ACM, New York (2002)

9. Vasalou, A., Hopfensitz, A., Pitt, J.V.: In praise of forgiveness: Ways for repairing trust breakdowns in one-off online interactions. Int. J. Human-Computer Studies 66, 466-480 (2008)

10. Villata, S.: Institutional social dynamic dependence networks. In: Boella, G., Pigozzi, G., Singh, M.P., Verhagen, H. (eds.) NORMAS, pp. 201-215 (2008) 